\title{
APPORTS DE LA TÉLÉDÉTECTION OPTIQUE MULTISOURCES DES PUITS PASTORAUX À LA CARTOGRAPHIE DES EAUX SOUTERRAINES DU SAHEL
}

\author{
Bernard Collignon \\ HYDROCONSEIL - 198, chemin d'Avignon - 84470 - Chateauneuf de Gadagne - \\ collignon@hydroconseil.com
}

\begin{abstract}
Résumé
Au Sahara et au Sahel, l'eau souterraine est une ressource limitée et précieuse. La vie quotidienne et le travail des éleveurs sont organisés en fonction de la localisation des points d'eau et de la profondeur de la nappe phréatique. Pour assurer le développement durable de ces régions, il est donc indispensable de construire des cartes piézométriques précises, y compris dans les zones les plus difficiles d'accès. Nous avons mis au point une méthode innovante permettant de dresser de telles cartes à partir de l'imagerie satellite en haute définition, afin de pouvoir compenser les lacunes de l'information hydrogéologique conventionnelle. L'artefact utilisé dans cette méthode est l'image des puits pastoraux.

Les pasteurs abreuvent leur bétail grâce à des puits profonds. Ils attellent des ânes ou des chameaux qui tirent l'eau avec une corde dont la longueur constitue une mesure précise de la profondeur de la surface piézométrique. Lors de l'opération, les bêtes et les cordes laissent sur le sol des traces que l'on peut observer et mesurer sur les images satellites, pour autant que leur résolution soit suffisante. Nous avons mis au point une technique d'interprétation de ces images qui permet (a) de repérer les puits pastoraux (sur images Landsat ou SPOT), (b) d'isoler les traces laissées par les bêtes utilisées pour tirer l'eau (sur images SPOT ou WorldView) et (c) d'estimer la profondeur de l'eau à partir de ces traces (sur images WorldView).

La méthode a été étalonnée soigneusement, par corrélation avec des mesures directes de la profondeur de l'eau dans les forages neufs. Après calibration, nous avons pu utiliser cette méthode pour dresser en quelques semaines la carte piézométrique de la dépression du Bornou au Nigeria $\left(20000 \mathrm{~km}^{2}\right)$. Cette zone est difficilement accessible par d'autres moyens à cause de l'insécurité qui prévaut dans cette région du Sahel depuis cinq ans. Nous avons ensuite testé la même méthode sur d'autres aquifères dans les pays du Sahel (Mauritanie, Sénégal, Mali, Burkina, Niger, Tchad et Soudan). Elle s'y applique très bien également, et il a ainsi été possible de cartographier en moins d'une année 1 million de $\mathrm{km}^{2}$ d'aquifère.
\end{abstract}

Mots-clés : Puits pastoraux - télédétection optique-- dépression piézométrique - nappe phréatique - Sahel - Nigeria - Bornou

\begin{abstract}
In Sahara and Sahel, groundwater is a limited and precious resource. The daily life and the work of the breeders are organized according to the location of wells and the depth of the water table. To manage the sustainable development of these regions, it is therefore essential to build accurate piezometric maps, even in the most difficult to access areas. We have developed an innovative method for producing such maps based on high-resolution satellite imagery, in order to compensate for the information gaps of conventional hydrogeological prospecting. The artifact used in this method is the image of pastoral wells. Breeders water their livestock using deep wells. To draw water, they harness donkeys or camels that pull water with a rope whose length is an accurate measure of the depth of the water table. During the operation, animals and ropes leave deep traces on the ground that can be observed and measured on satellite images, provided that their resolution is sufficient. We have developed a technique for interpreting these images that allows (a) to locate pastoral wells (on Landsat or SPOT images), (b) to isolate the traces left by the animals drawing water (on SPOT or WorldView images) and (c) to estimate the water depth from these traces (on WorldView images).

The method was carefully calibrated using correlation with direct measurements of water depth in new boreholes. After calibration, we were able to use this remote sensing technique to draw up in a few weeks the piezometric map of a large aquifer depression in Nigeria (Bornu - 20,000 km²). This area is difficult to access by other means because of the insecurity that has prevailed in these regions of the Sahel for the past 5 years. We then tested the same method on other aquifers in other Sahelian countries (Mauritania, Senegal, Mali, Burkina, Niger, Chad and Sudan). The method also proved to be very effective and we were able to map 1 million $\mathrm{km}^{2}$ of aquifer in less than a year.
\end{abstract}

Keywords: Pastoral wells - optical remote sensing- water table - unconfined aquifer - Sahel - Nigeria - Bornu 


\section{Problématique}

Depuis 2015, Hydroconseil a supervisé deux grandes campagnes de forage financées par l'Union européenne dans la partie septentrionale du Sahel tchadien : le programme d'alimentation en eau potable et d'assainissement (PAEPA - 1100 forages) et le programme résilience et emploi au Lac Tchad (RESTE 210 forages).

Ces forages ont été utilisés pour dresser la carte piézométrique de plusieurs zones. Cependant, nous manquions encore de mesures piézométriques dans les zones pastorales de la frange nord du bassin du Lac Tchad, car les campagnes de forage d'eau potable se sont déroulées dans les zones du centre et du sud du bassin, où sont concentrées les populations sédentaires.

Les campagnes de forage ont peu couvert les zones septentrionales du bassin parce que ce sont des zones de nomadisme pastoral où il $y$ a très peu de villages permanents. Les prélèvements d'eau souterraine dans cette zone sont néanmoins importants. Le plus souvent, cependant, l'eau n'est pas extraite d'un forage moderne mais de puits profonds de gros diamètre creusés dans les sables et les grès. La majorité de ces ouvrages sont réalisés par des puisatiers traditionnels qui relèvent $\mathrm{du}$ marché informel (Collignon \& Debris, 1994). Pour cette raison, ces ouvrages sont mal recensés dans les bases de données à partir desquelles les cartes piézométriques conventionnelles sont construites.

Il est particulièrement difficile et coûteux de réaliser des campagnes de mesure dans ces régions, car elles sont enclavées, les pistes sont difficiles (dunes) et certaines régions (Bornou, Kadzell, Kanem, Lac) sont très mal sécurisées (il s'agit de la zone où sévit l'organisation Boko Haram).

Ces informations lacunaires rendent délicate la gestion durable des ressources en eaux souterraines car, sans une bonne carte piézométrique, il est impossible d'établir le bilan en eau de l'aquifère et d'évaluer sa recharge par les précipitations. Or ces informations ont une importance capitale pour orienter les stratégies de développement régional et répondre à une question fondamentale : les eaux souterraines sont-elles suffisamment abondantes pour accompagner l'augmentation des prélèvements que laisse prévoir la croissance de la population et du cheptel?

L'objectif de cette recherche est donc d'établir la carte piézométrique des zones qui entourent le lac Tchad (Bornou, Kadzell, Kanem, Barh el Ghazal, Hadjer Lamis) en exploitant les images satellitaires en complément des levés piézométriques traditionnels.

\section{Les puits pastoraux}

\subsection{L'importance de l'élevage pastoral}

Le Sahel est caractérisé par un climat aride à semi-aride (100 à $500 \mathrm{~mm}$ de pluie par an, avec de fortes variations interannuelles). L'agriculture ne s'étend guère au-delà des cultures de décrue dans les dépressions inter-dunaires. Cette région est cependant assez densément peuplée et elle est régulièrement parcourue par différents groupes de pasteurs qui nomadisent sur de grandes étendues (Arabes, Kredas, Dazas, Peuls Mbororos, Boudoumas...) et ont mis au point un mode de vie et de production très bien adapté à cet écosystème particulier (Bazin et al. 2013). La subsistance de ces populations repose sur l'élevage transhumant des ruminants. Celui-ci a pour but d'optimiser l'exploitation de ressources fourragères, très variables dans le temps et dans l'espace (Diawara et al., 2014), à partir desquelles les hommes ont développé un système de production efficace qui permet de bien valoriser la biomasse disponible dans les zones arides (Scoones, 1995)

L'effectif du cheptel présent dans cette région est énorme. En 2016, le ministère de l'Élevage a recensé 21 millions de têtes de ruminants dans les trois provinces nordsahéliennes du Tchad (Tableau 1). Dans ces zones, les besoins en eau du bétail sont ainsi considérablement plus élevés que les besoins pour l'alimentation en eau potable de la population ( 2 millions d'habitants dans la même zone en 2016).

La pérennité du mode de production des pasteurs repose sur l'exploitation de centaines de puits dispersés à travers les zones de pâturage. Cette stratégie permet de valoriser au mieux la ressource fourragère ainsi que d'éviter de trop fortes concentrations de bétail, lesquelles entraineraient une dégradation du couvert végétal par surpâturage autour des points d'eau (Cesaro et al., 2014) et (Valenza, 1981). Quelques forages motorisés et des puits en ciment ont été construits par le gouvernement depuis 20 ans. Ceux-ci ne constituent néanmoins qu'un appoint, car ils sont beaucoup moins nombreux que les puits artisanaux (Langlais et al., 2012).

\begin{tabular}{|l|c|c|c|c|c|c|}
\hline \multicolumn{7}{|c|}{ bovins ovins caprins camelins equins asins } \\
\hline Batha & 4269 & 3274 & 3000 & 913 & 114 & 228 \\
\hline Kanem & 1745 & 1988 & 2108 & 468 & 100 & 245 \\
\hline Bahr el Gazal & 747 & 720 & 815 & 290 & 47 & 126 \\
\hline \multicolumn{4}{|c|}{} \\
\cline { 2 - 7 } Total & 6761 & 5982 & 5923 & 1671 & 261 & 599 \\
\cline { 2 - 7 } & & 21197 \\
\hline
\end{tabular}

Tableau 1 : Effectifs du cheptel dans la zone d'étude (en milliers de têtes) 


\subsection{Les puits pastoraux}

Au Sahel et au Sahara, les puits utilisés pour abreuver le bétail ont une profondeur qui varie très fortement en fonction de la topographie et des conditions hydrogéologiques locales. Les moins profonds sont de simples puisards de quelques mètres, creusés dans les bas-fonds inondables et qui sont reconstruits après chaque saison des pluies. Les plus profonds sont des ouvrages impressionnants, qui dépassent parfois 100 mètres de profondeur. Les images satellites de ces puits profonds offrent la possibilité de mesurer la profondeur de la surface piézométrique.

Autour du lac Tchad (dans les régions du Bornou, du Kanem, du Lac, du Hadjer Lamis...), ces puits profonds sont, pour la plupart, creusés dans les formations sédimentaires (sables et grès) du Pliocène et du Quaternaire. Ils sont creusés à la main par des entrepreneurs puisatiers, rémunérés par les villageois et les éleveurs. Le cuvelage des puits traditionnel est étançonné en bois ou en pierre, tandis que celui des puits modernes l'est en béton armé. Dans les deux cas, la hauteur d'eau en fond de puits est limitée à quelques mètres, à cause des moyens d'exhaure très limités dont disposent les puisatiers pour creuser sous le niveau de l'eau (Collignon \& Debris, 1994), (Langlais et al., 2014).

\subsection{L'exhaure animale au Sahara et au Sahel}

Au Sahel, seule une petite minorité de puits pastoraux sont motorisés. Dans plus de $95 \%$ des puits, l'eau est extraite par exhaure animale. L'exhaure est réalisée par les éleveurs eux-mêmes, en utilisant des ânes, des chevaux et des chameaux. Elle est réalisée en attelant une ou plusieurs bêtes à une longue corde au bout de laquelle est accrochée une outre en cuir ou en bâche plastique d'un volume de 50 à 80 litres (Photos 1 et 2). La corde passe sur une poulie en bois, disposée à la verticale du puits. Cette poulie est accrochée soit à un portique métallique (pour les puits modernes au cuvelage cimenté), soit à une fourche en bois (pour les puits traditionnels) (Langlais et al., 2014). Chaque éleveur qui vient puiser de l'eau atèle une ou plusieurs de ses bêtes et tire l'eau avec une corde dont la longueur est égale à la profondeur de l'eau dans le puits (Figure 1). La longueur de la corde équivaut donc à ce que les hydrogéologues appellent le niveau dynamique de l'aquifère, qui varie de quelques dizaines de centimètres au cours de la journée, en fonction de la fréquentation du puits par les troupeaux.

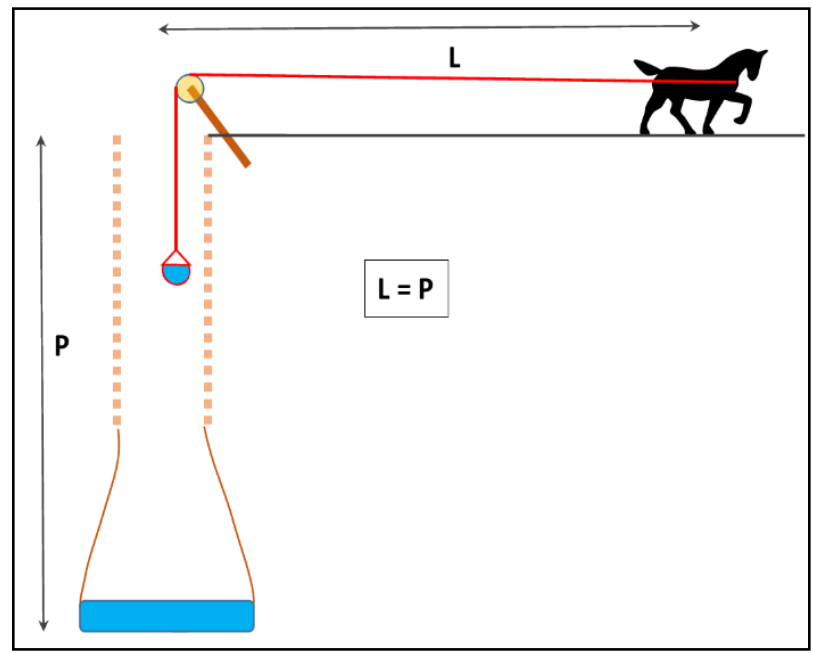

Figure 1 : L'exhaure animale dans les puits profonds.

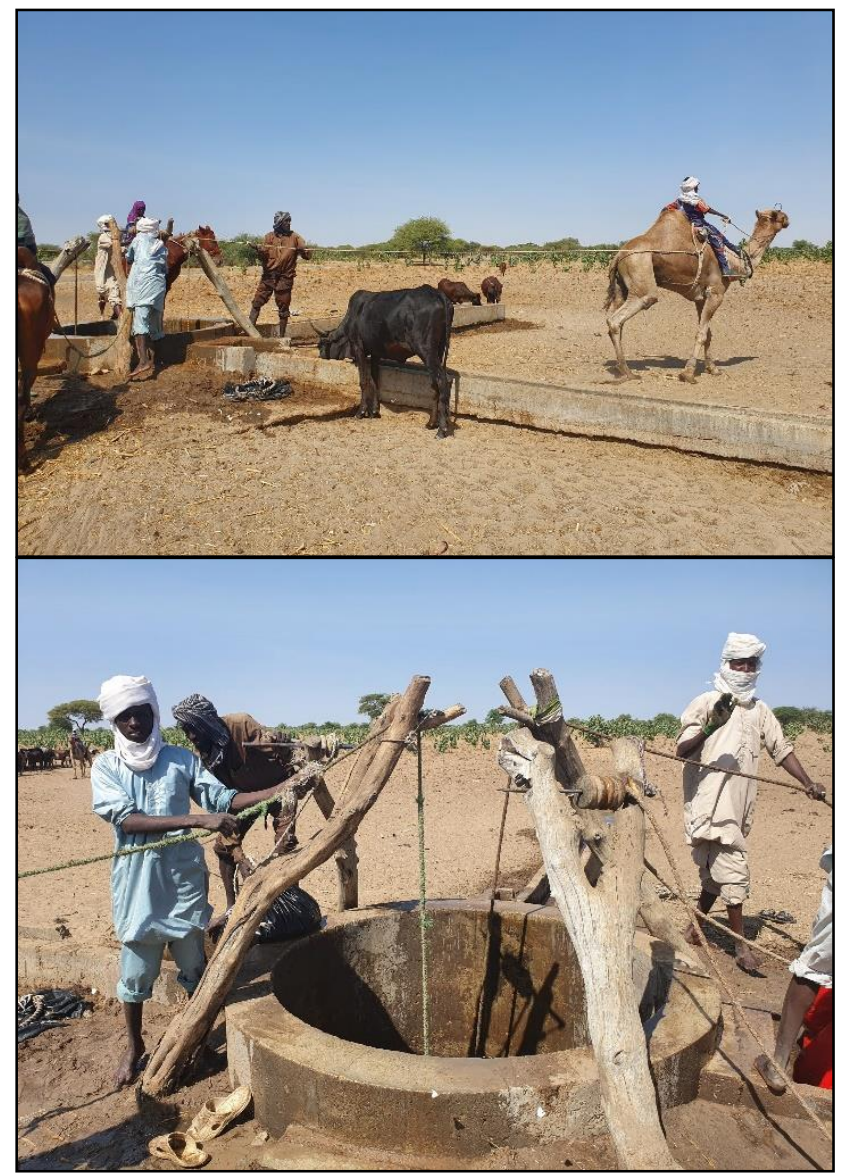

Photos 1 et 2: Exhaure avec un chameau (haut) et fourches d'exhaure traditionnelles en bois d'acacia (bas) dans la région du Hadjer Lamis, au Tchad (photos Ibrahim A.Nassour). 


\section{Une méthodologie innovante pour élaborer des cartes piézométriques}

Nous avons mis au point une méthodologie innovante qui exploite les traces laissées lors de l'exhaure, afin de pouvoir construire des cartes piézométriques à partir des images satellites (Collignon, 2020a et Collignon, 2020b).

\subsection{Etape 1 - Repérer les sites de puits pastoraux sur images satellites}

Les puits pastoraux peuvent être repérés sur les images satellite par les traces que laissent les troupeaux qui convergent vers les puits. Ces traces sont généralement rayonnantes (Figure 2), car il s'agit d'une libre pâture, dans des terres qui ne sont pas clôturées. Pour les puits les plus fréquentés, la longueur des traces peut atteindre $5 \mathrm{~km}$ et elles se marquent par des bandes claires de quelques dizaines de mètres de large. Elles correspondent à la forte réflectance du sol sableux ameubli lors du passage du troupeau et érodé par le vent.

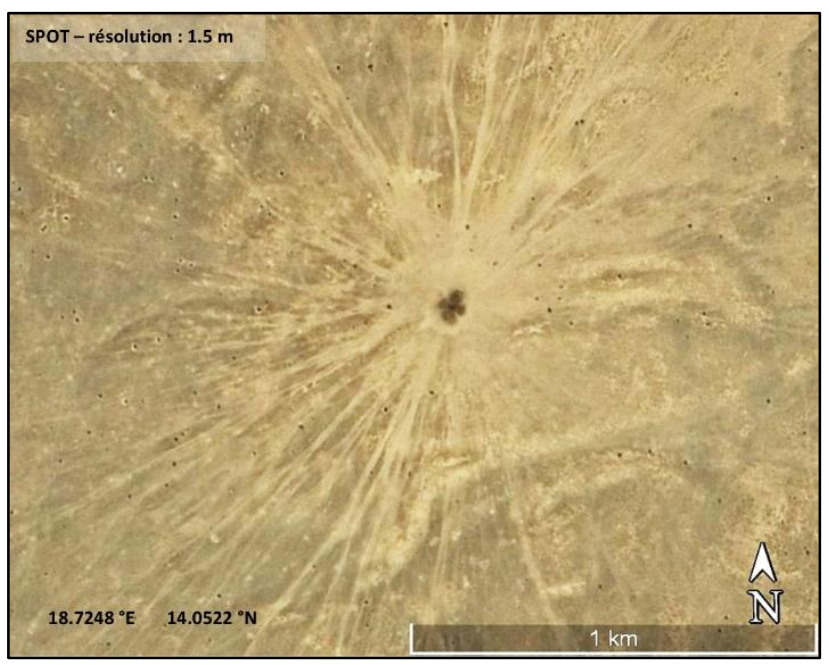

Figure 2 : Traces de passage du bétail permettant de localiser les puits pastoraux (source : Google Earth)

\subsection{Etape 2 - Repérer les puits eux-mêmes, grâce à la signature spectrale des déjections des ruminants}

$\mathrm{Au}$ centre des traces rayonnantes, on peut presque toujours repérer sur les images satellites une zone plus sombre, de 40 à 150 mètres de diamètre. Cette zone correspond à l'accumulation des déjections des ruminants qui patientent avant d'aller s'abreuver. Les alentours des puits constituent ainsi des lieux de concentration de matière organique (Diawara et al., 2014). Les excrétas, mélangés avec le sable, forment des couches épaisses (dépassant parfois un mètre). La signature spectrale de cette couche est très forte: elle a une très faible réflectance, aussi bien dans le spectre visible que dans le proche infrarouge (Girard \& Girard, 1999).
La marque sombre de la matière organique constitue un marqueur très stable des puits pastoraux, car elle subsiste d'une année à l'autre, y compris pendant les mois où il n'y a pas de bétail, et ce pendant plusieurs années (Figure 3 et Photo 4). Dans de nombreux cas, sa forme évoque un trèfle à quatre feuilles de quelques dizaines de mètres de diamètre.

Les bandes claires qui séparent les zones sombres correspondent aux traces de passage des animaux qui font un mouvement de va-et-vient autour du puits pour en tirer des outres d'eau.

Les traces d'exhaure ont toujours une meilleure réflectance dans le spectre visible que les zones environnantes. Ceci correspond vraisemblablement à la signature du sable siliceux régulièrement remué par le passage des animaux alors que les particules plus sombres (matière organique) sont déblayées par le vent parce qu'elles sont plus légères et qu'elles ont une forme allongée qui favorise le transport éolien.

Le contraste entre les traces d'exhaure et les zones qui les entourent est d'autant plus net que le puits est beaucoup fréquenté. En effet, les animaux qui attendent leur tour pour venir boire produisent de la matière organique (sombre) tout en restant à l'écart de la bande étroite dans laquelle circulent les animaux utilisés pour le puisage.

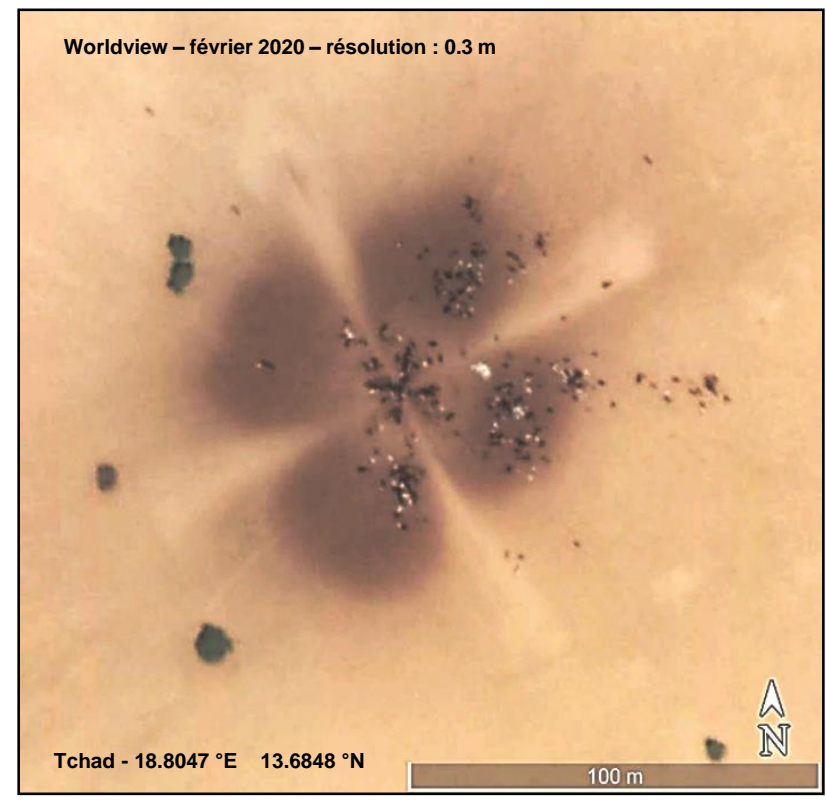

Figure 3 : Image satellite caractéristique d'un puits pastoral du Batha ou du Bahr el Ghazal (source : Google Earth) 


\subsection{Etape 3 - Mesurer la longueur des traces d'exhaure sur les images satellites}

Les bêtes utilisées pour l'exhaure suivent un parcours très régulier, parfaitement défini, qui a la forme d'une goutte d'eau allongée (Figure 3, Figure 4 et Figure 5). Elles commencent par s'éloigner du puits en ligne droite, jusqu'à ce que l'outre contenant l'eau arrive à la surface. Le berger détache alors la corde, et l'animal décrit un demi-cercle de quelques mètres de rayon avant de revenir au puits en ligne droite. Le long de cette trace, le sable, régulièrement remué par les animaux, est fragilisé et érodé par le vent, et sa réflectance est élevée.

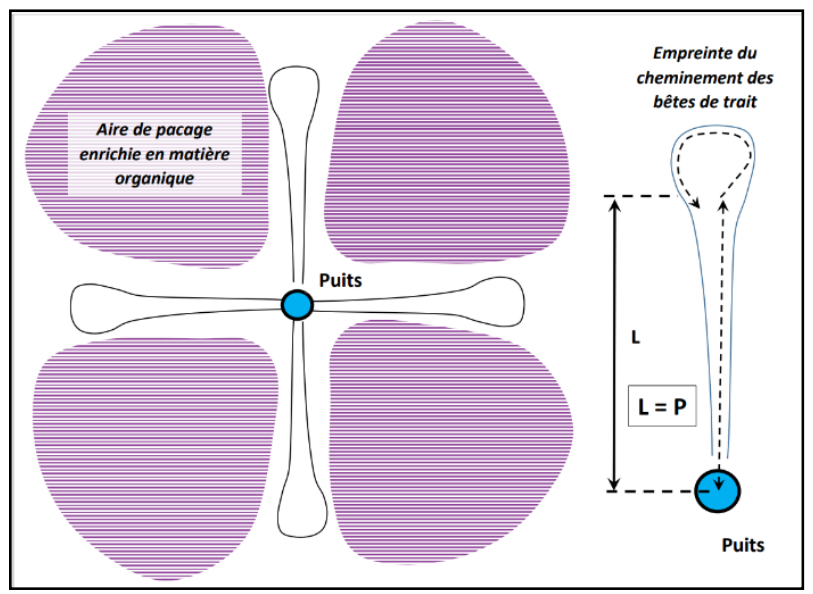

Figure 4 : Interprétation des images satellitaires

On observe autour de chaque puits une série de traces semblables (de un à huit, le nombre le plus fréquent étant quatre, qui correspond au nombre des poulies disposées à angle droit sur un portique métallique).

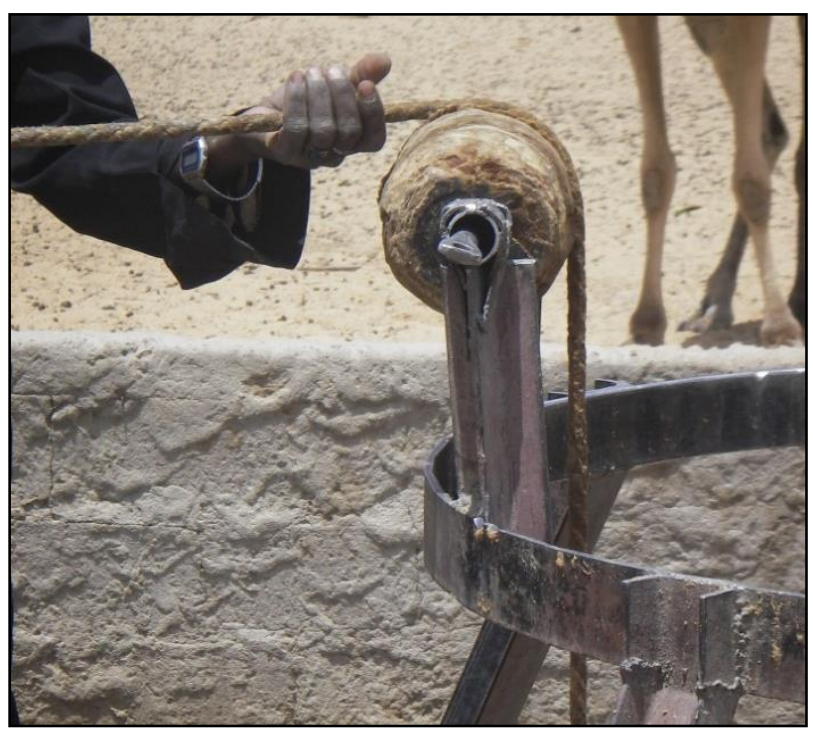

Photo 3. Poulie en bois utilisée par les bergers au Sahel (photo Roland Tapia).

Photo 4. Accumulation de matière organique sombre autour d'un puits dans le massif de Termit au Niger (photo Roland Tapia).

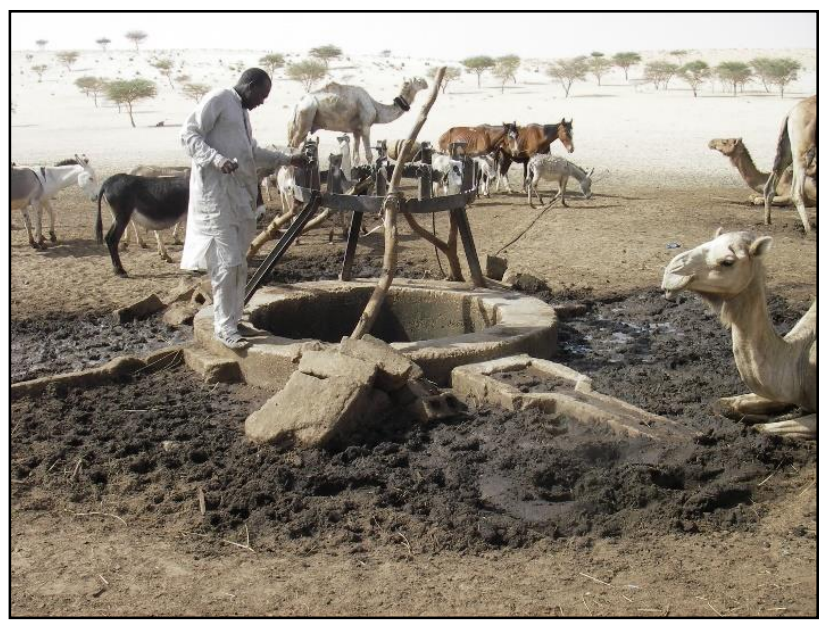

Le repérage des puits pastoraux sur les images satellites est une opération délicate, qu'il est préférable de réaliser sur des images à haute résolution.

Avec un peu d'entraînement, on peut localiser les puits sur des images avec 30 mètres de résolution, mais l'identification des traces d'exhaure est impossible avec une telle résolution.

Avec des images haute résolution, il est généralement possible d'identifier les traces rayonnantes convergeant vers le puits. On peut même parfois identifier les traces d'exhaure, mais leur mesure précise est difficile.

En revanche, les images ayant une très haute résolution (meilleure que $0.5 \mathrm{~m}$ ) permettent de mesurer la longueur des traces d'exhaure avec une incertitude de 2 à 3 mètres.

Dans le cadre de l'étude de l'aquifère Plio-quaternaire du bassin du lac Tchad, nous avons recherché systématiquement les puits pastoraux sur les images diffusées via Google Earth Pro ${ }^{\circledR}$. Nous en avons localisé en quelques jours plus de 700 , rien que dans la région du Bornou, au Nigeria. L'analyse des images satellites est donc un outil efficace pour repérer les puits pastoraux dans les zones sahéliennes.

Nous avons recherché systématiquement les traces d'exhaure animale sur ces ouvrages et nous avons pu les identifier et les mesurer dans la majorité des cas (pour 83\% des puits repérés, ce qui représente 620 mesures de profondeur d'eau). 
Ce travail a été réalisé par analyse directe des images, ce qui est assez fastidieux. II serait évidemment intéressant d'automatiser la procédure de recherche et de mesure, mais une telle opération soulève des difficultés, car l'intensité des marqueurs varie beaucoup en fonction du sol (argileux ou sableux), de la saison de prise de vue (sèche ou humide) et même de l'heure de passage du satellite (jour ou nuit). De plus, les différents marqueurs des puits pastoraux sur les images n'ont pas la même rémanence :

- la zone enrichie en matière organique est un marqueur fort, avec une rémanence d'une dizaine d'années (les dépôts sont encore visibles pendant plusieurs années après l'abandon du puits) ;

- les traces rayonnantes vers le puits sont également un marqueur fort, avec une rémanence de quelques années ; leur intensité dépend de deux facteurs : (a) la fréquentation du puits et (b) la nature du sol (elles sont davantage visibles là où subsiste une végétation herbacée, labourée par le passage des troupeaux) ;

- les chemins de puisage sont des marqueurs plus fragiles, qui disparaissent au bout de quelques mois en cas d'abandon du puits ou de mise en culture de la zone environnante.

\subsection{Les images satellites les plus adaptées}

Nous avons réalisé ce travail à partir d'images directement accessibles sur Google Earth Pro ${ }^{\circledR}$. Les images sources varient selon les zones. Cela permet de comparer les performances de la méthode de télédétection suivant la résolution des images (Tableau 2 et Figure 5).

Les images en basse résolution (satellites Landsat 7 et 8) sont utiles pour repérer rapidement une partie des puits sur de très vastes superficies, grâce aux traces de circulation du bétail, rayonnantes autour des puits.

En revanche, ces images ne permettent pas de discerner les traces d'exhaure animale et d'utiliser cette méthode pour évaluer la profondeur de la surface piézométrique (Figure 5 - haut).

La perception des traces d'exhaure est rendue possible par les images en haute résolution (satellites SPOT 6 et 7 et surtout WorldView 3 et 4). On peut alors mesurer les traces d'exhaure et en déduire la profondeur de la surface piézométrique. La longueur à prendre en considération est la distance qui sépare le centre du puits (à l'intersection des différentes traces d'exhaure) de l'endroit où l'animal commence à tourner, quand il a été libéré de sa charge (Figures 4 et 5).

Figure 5 : Visualisation de puits pastoraux d'une même zone (Tchad - Batha Ouest) sur trois images satellites de résolution croissante et une photo aérienne (source: Google Earth).
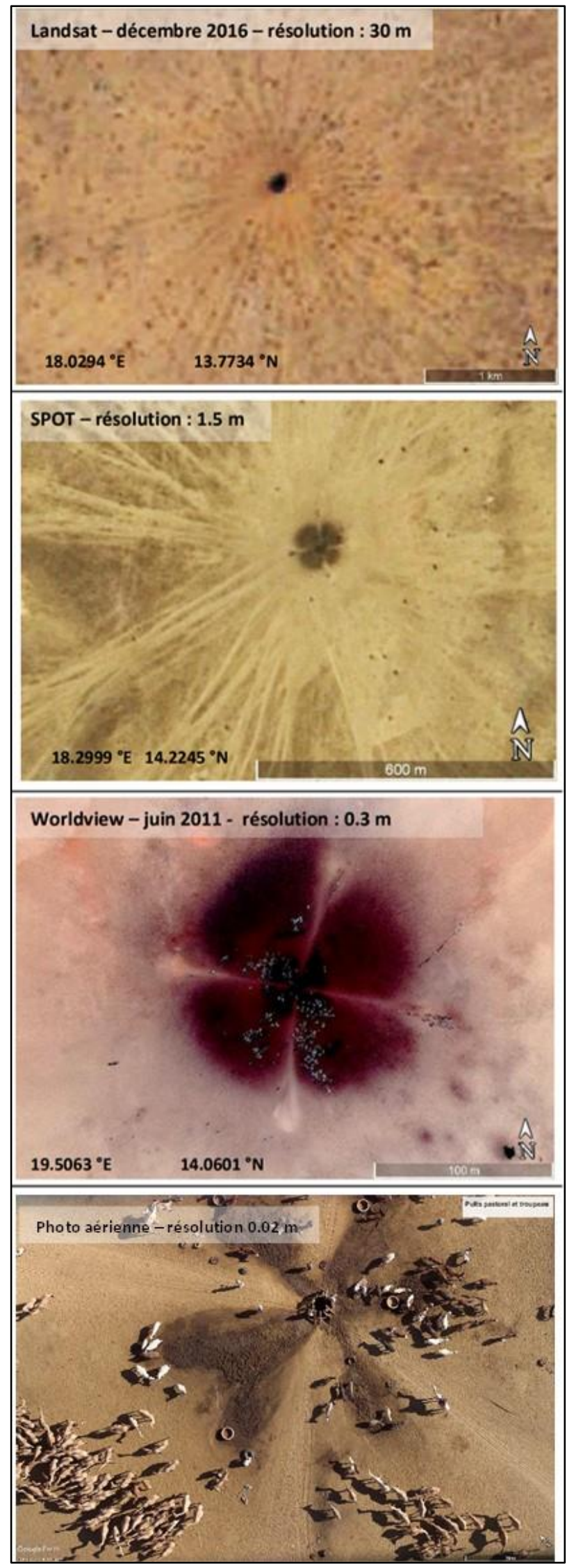

Revue Française de Photogrammétrie et Télédétection Volume 223 (année 2021) numéro Spécial Afrique https://doi.org/10.52638/rfpt.2021.602 


\begin{tabular}{|r|c|c|c|}
\hline Résolution & $\begin{array}{c}\text { Landsat } \\
7 \text { et } 8\end{array}$ & $\begin{array}{c}\text { SPOT } \\
6 \text { et } 7\end{array}$ & $\begin{array}{c}\text { Worldview } \\
3 \text { et } 4\end{array}$ \\
\hline $\begin{array}{r}\text { Traces } \\
\text { rayonnantes }\end{array}$ & $\begin{array}{c}\text { Souvent } \\
\text { discernables sur } \\
\text { les puits très } \\
\text { fréquentés }\end{array}$ & $\begin{array}{c}1.5 \mathrm{~m} \\
\text { Toujours } \\
\text { discernables }\end{array}$ & $\begin{array}{c}\text { Toujours } \\
\text { discernables }\end{array}$ \\
\hline $\begin{array}{r}\text { Auréole de } \\
\text { matière }\end{array}$ & $\begin{array}{c}\text { Discernables, } \\
\text { mais non } \\
\text { mesurables }\end{array}$ & $\begin{array}{c}\text { Souvent bien } \\
\text { visibles et } \\
\text { mesurables }\end{array}$ & $\begin{array}{c}\text { Toujours bien } \\
\text { visibles et } \\
\text { mesurables }\end{array}$ \\
\hline $\begin{array}{r}\text { Traces } \\
\text { d'exhaure }\end{array}$ & Jamais visibles & $\begin{array}{c}\text { Souvent visibles } \\
\text { et parfois } \\
\text { mesurables }\end{array}$ & $\begin{array}{c}\text { Toujours visibles } \\
\text { et mesurables; } \\
\text { bétail } \\
\text { dénombrable }\end{array}$ \\
\hline
\end{tabular}

Tableau 2 : Potentiel de la méthode par télédétection des puits pastoraux, en fonction de la résolution des images

La même méthode pourrait être utilisée avec des photos aériennes (Figure 5-bas). Encore faudrait-il disposer d'une couverture générale des pays du Sahel, qui n'existe pas encore.

\section{Etalonnage de la méthode (vérité terrain)}

Pour valider une méthode d'investigation basée sur des images satellites, il est indispensable de comparer les valeurs estimées de cette façon avec des valeurs mesurées directement sur le terrain, qui serviront à la fois de calage et de validation (Girard \& Girard, 1999).

Pour mesurer la profondeur de la surface piézométrique, nous disposons d'une méthode simple et fiable: la mesurer directement à l'intérieur d'un puits ou d'un forage au repos (sans pompage). Dans le cas des zones pastorales, l'usage de cette méthode est cependant rendue compliqué par deux facteurs : (a) un grand nombre de puits pastoraux sont situés dans des dépressions, au milieu de champs de dunes, et ne sont pas accessibles avec des véhicules motorisés et (b) pour obtenir une mesure fiable du niveau piézométrique, il faudrait arrêter le puisage de l'eau pendant plusieurs heures, ce qui est difficile dans une région où les troupeaux se succèdent sans discontinuer aux points d'eau. Quant aux mesures nocturnes, elles sont difficilement envisageables dans le contexte actuel d'insécurité,

Nous avons quand même pu étalonner la méthode, en couplant l'analyse des images satellites avec des mesures directes du niveau de l'eau dans les nouveaux forages réalisés dans le cadre du programme PAEPA, avant l'installation des pompes.
La Figure 6 montre que les deux méthodes de mesure du niveau de l'eau donnent des résultats comparables, avec un écart quadratique moyen de moins de 3 mètres, ce qui est tout-à-fait suffisant pour dresser des cartes piézométriques régionales. La corrélation entre les deux types de mesure est excellente. Les images satellites de traces d'exhaure constituent donc un indicateur fiable de la profondeur de l'eau.

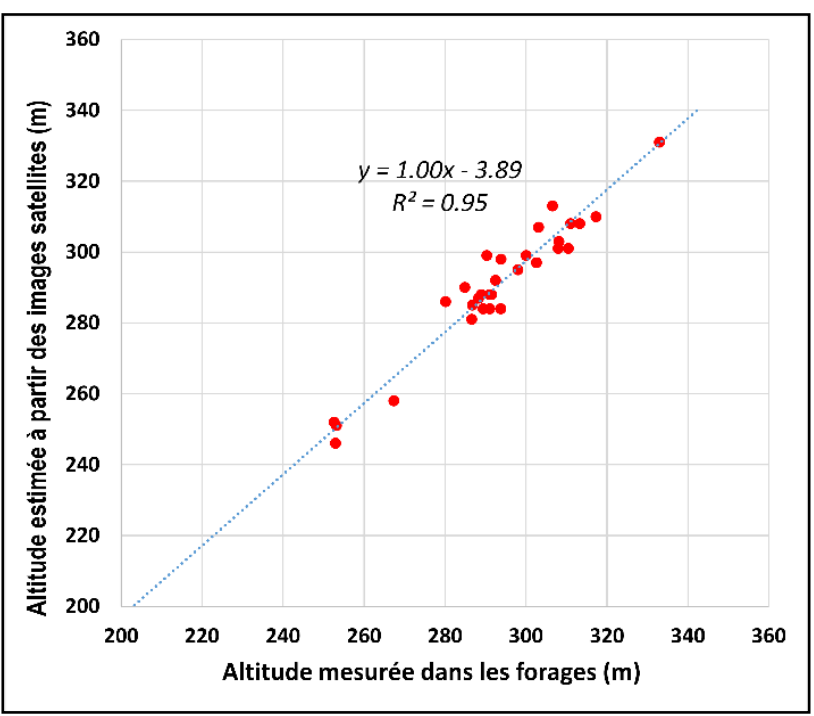

Figure 6 : Test de validation de la méthode par télédétection des puits pastoraux, avec les altitudes piézométriques mesurées directement dans les forages.

\section{Construction d'une carte piézométrique à partir des images satellites}

Pour caractériser la circulation des eaux souterraines dans un aquifère, l'outil de base de l'hydrogéologue est la carte piézométrique, c'est-à-dire la carte de l'altitude de la surface libre de l'eau dans les puits laissés au repos.

Nous allons l'illustrer avec la nappe phréatique dans la région du Bornou, au nord-est du Nigeria, près du lac Tchad. Une ancienne carte piézométrique (Figure 7 - haut) met en évidence une dépression d'environ $10,000 \mathrm{~km}^{2}$, c'est-à-dire une zone où la surface libre de l'aquifère est déprimée de 50 mètres par rapport à toutes les zones environnantes. Cette dépression a des conséquences pratiques : elle oblige les villageois à creuser des puits beaucoup plus profonds qu'ailleurs. Cette zone déprimée indique un bilan en eau négatif. La forte évapotranspiration concentre les sels dissous dans les eaux souterraines et celles-ci sont plus salées au centre de la dépression (Zairi, 2008).

La nouvelle carte piézométrique (Figure 7 - bas) a été construite par télédétection, en utilisant uniquement la mesure des traces d'exhaure animale autour de 620 puits agro-pastoraux, sur une zone couvrant la dépression 
décrite ci-dessus et en étendant la couverture encore plus loin vers l'ouest (jusqu'au méridien $10.5^{\circ} \mathrm{E}$ ). Cette carte est beaucoup plus complète, plus précise et plus détaillée que la précédente, et que la plupart des cartes piézométriques réalisées antérieurement dans la région. Elle met en évidence les zones hautes, où la nappe est rechargée par les fleuves Komadougou et Komadougou Yobe et par le lac Tchad. La dépression piézométrique du Bornou se situe entre la Komadougou et la ville de Maidiguri.

Cette carte plus détaillée montre que la dépression piézométrique du Bornou descend jusqu'à la cote $230 \mathrm{~m}$ a.s.l. aux alentours de la ville de Gubio. Elle s'étend plus loin vers l'ouest que ce qui était connu (jusqu'au méridien $11^{\circ} \mathrm{E}$ ) et on constate qu'elle est divisée en deux par un bourrelet allongé qui culmine à la cote 260 .

Sur une carte piézométrique, ce genre de bourrelet constitue la marque d'une zone de recharge de l'aquifère. II est remarquable que cette zone de recharge suive exactement le tracé d'un affleurement que les géologues connaissent bien : la crête dunaire de Bama (Zarma et Turku ; 2015). Celle-ci est constituée par une série de petites dunes sableuses parallèles qui marquent un ancien rivage du lac Tchad. La nouvelle carte piézométrique construite à partir des images satellites suggère que ces dunes parallèles constituent une zone préférentielle de recharge de la nappe phréatique.

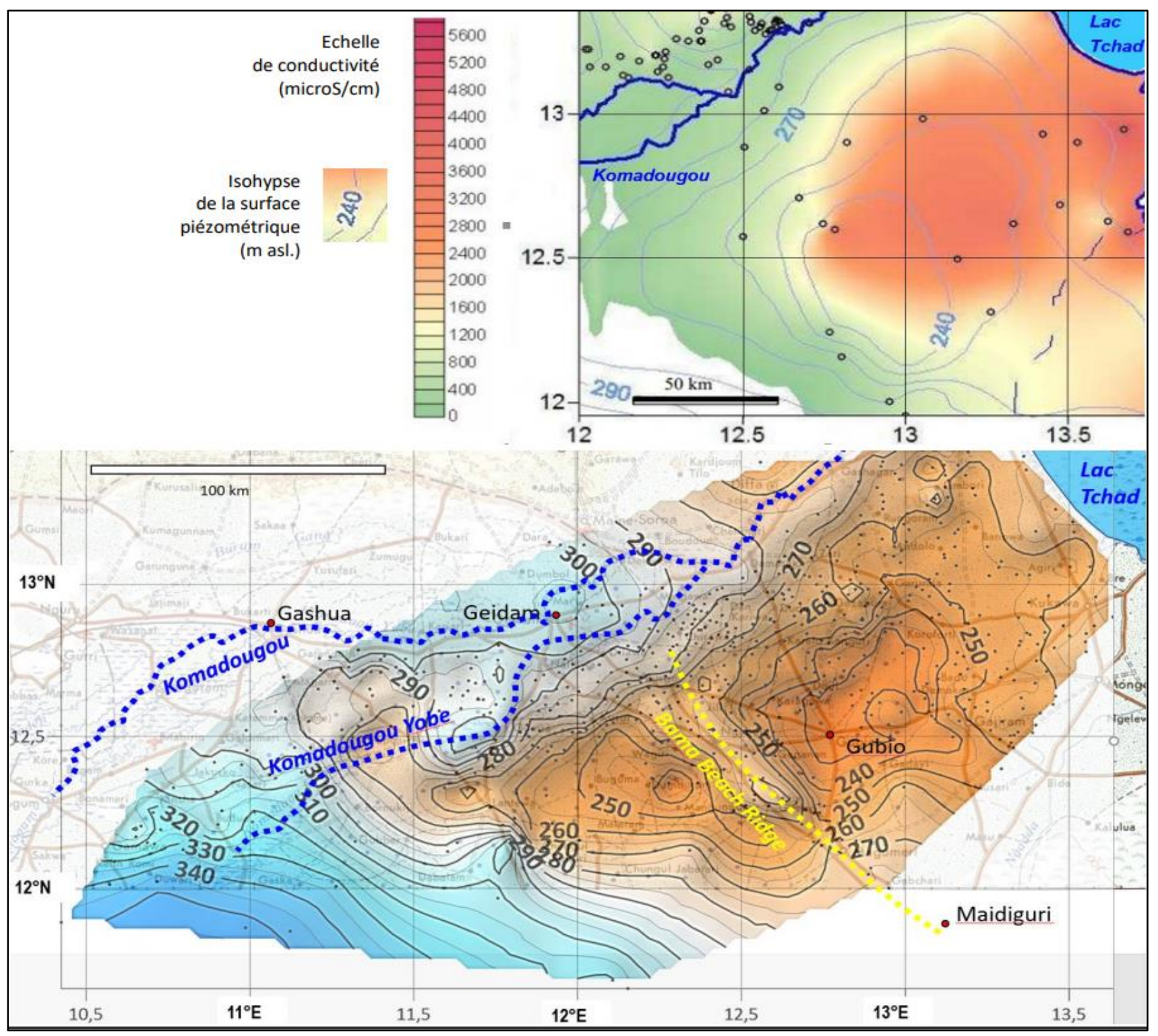

Figure 7 : Application de la méthode par télédétection des puits pastoraux pour actualiser, étendre et améliorer la carte piézométrique de la dépression du Bornou (nord du Nigeria). Haut : carte basée sur des mesures directe dans les forages (Zairi, 2008). Bas : carte construite à partir des traces d'exhaure repérées sur images satellites. 


\section{Conclusion : potentiel et limites de la méthode}

\subsection{Zone d'application de la méthode}

La méthode par télédétection des puits pastoraux est particulièrement bien adaptée aux régions du Sahel et du Sahara (Figure 9).

Dans ces régions, elle s'applique dès que prédomine l'exhaure animale, c'est-à-dire là où la profondeur de l'eau est supérieure à 20 mètres. Près de $90 \%$ de ces puits ont entre 30 et 80 mètres de profondeur dans le Bornou.

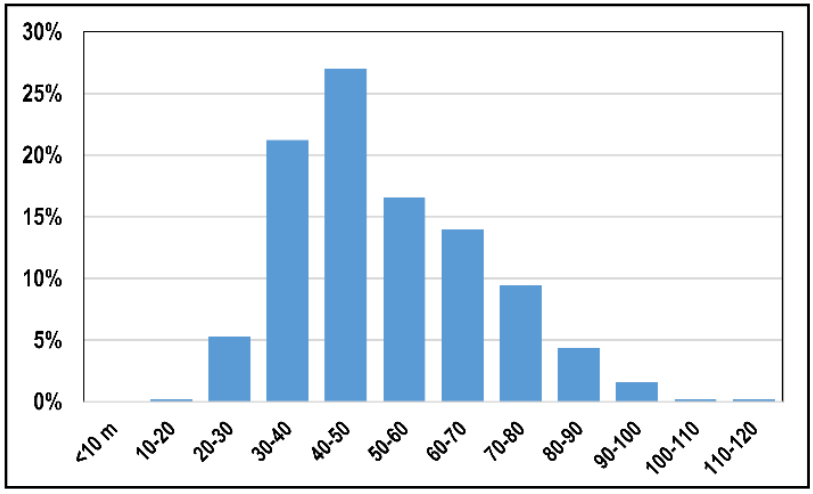

Figure 8: Distribution statistique de la profondeur des puits à exhaure animale dans le Bornou (Nigeria)

Nous avons ainsi pu appliquer la méthode de cartographie par télédétection des puits pastoraux avec succès sur $60 \%$ de l'étendue des grands aquifères sédimentaires du Sahel (entre les isohyètes 100 et $500 \mathrm{~mm} / \mathrm{an}$ ), soit plus de 1 million de $\mathrm{km}^{2}$ (Figure 9). Seules les zones où la profondeur de l'eau est inférieure à 20 mètres échappent à ce type d'analyse (Figure 8).

Nous avons appliqué la même méthode pour estimer l'évolution pluriannuelle de la piézométrie, en exploitant des couples d'images accessibles via la fonction « images d'archives » de Google Earth. Bien que ces images soient disparates, il est souvent possible de comparer la longueur des traces d'exhaure à différentes époques. Nous avons réalisé ce travail pour quatre aquifères (Bornou, Trarza, lullemeden CT et lullemeden $\mathrm{Cl}$ ) et l'analyse statistique de ces mesures indique que l'évolution saisonnière ou pluriannuelle de ces quatre aquifères est inférieure à l'incertitude de mesure (travaux en cours).

\subsection{Limites de la méthode par télédétection}

Quand l'eau est très peu profonde, la méthode ne s'applique pas, car les bergers préfèrent puiser l'eau directement à la main et il n'y a pas de traces d'exhaure animale.
La longueur des traces observées sur les images satellitaires présente l'intérêt d'être une moyenne pondérée de nombreuses traces, et donc d'intégrer différents états de la nappe que l'on ne pourrait pas mesurer lors d'une seule campagne piézométrique. Cela concerne en particulier la différence de niveau entre le matin (nappe haute) et le soir (nappe basse). En revanche, cette mesure ne représente que le niveau de la nappe pendant la saison de pâture (juillet à janvier) et non en saison sèche, quand les troupeaux ont migré vers le sud, dans le Salamat, le Hadjer Lamis, le Guera (Bazin, Bechir Ali, \& Khamis Djibrine, 2013). Cette méthode n'est donc pas adaptée pour suivre le battement saisonnier de la nappe.

\subsection{Puissance de cette méthode par télédétection des puits pastoraux}

L'exemple du Bornou (Nigeria) démontre qu'il est possible de construire des cartes piézométriques de la nappe phréatique à partir de l'interprétation des images satellites dans les zones d'élevage pastoral, là où prédomine l'exhaure animale.

Une méthode comparable a déjà été utilisée au Niger pour établir la carte piézométrique de la région de l'Azawagh, en mesurant la profondeur de 320 puits (Langlais, 2017).

L'utilisation de cette méthode est facilitée par l'existence de grands troupeaux, qui induisent une forte production de matière organique qui permet de repérer les puits grâce à la faible réflectance de la matière organique accumulée autour d'eux.

Cette méthode par télédétection ne se substitue pas aux mesures directes de niveau piézométrique sur le terrain, mais elle permet d'étendre très significativement la couverture des cartes dans toutes les zones du Sahel et du Sahara où les coûts des campagnes de mesure de terrain sont très élevés (à cause des contraintes logistiques) et où l'accès à de larges parts des zones pastorales est devenu très difficile depuis cinq ans, à cause de l'insécurité (Figure 9).

La télédétection permet ainsi d'établir en quelques semaines une carte piézométrique à l'échelle régionale sur des zones de plusieurs centaines de milliers de $\mathrm{km}^{2}$.

De plus, l'analyse diachronique, réalisée à partir d'images prises à plusieurs années d'intervalles, ouvre des perspectives très intéressantes pour étudier l'évolution de ces aquifères dans un contexte de changement climatique. 


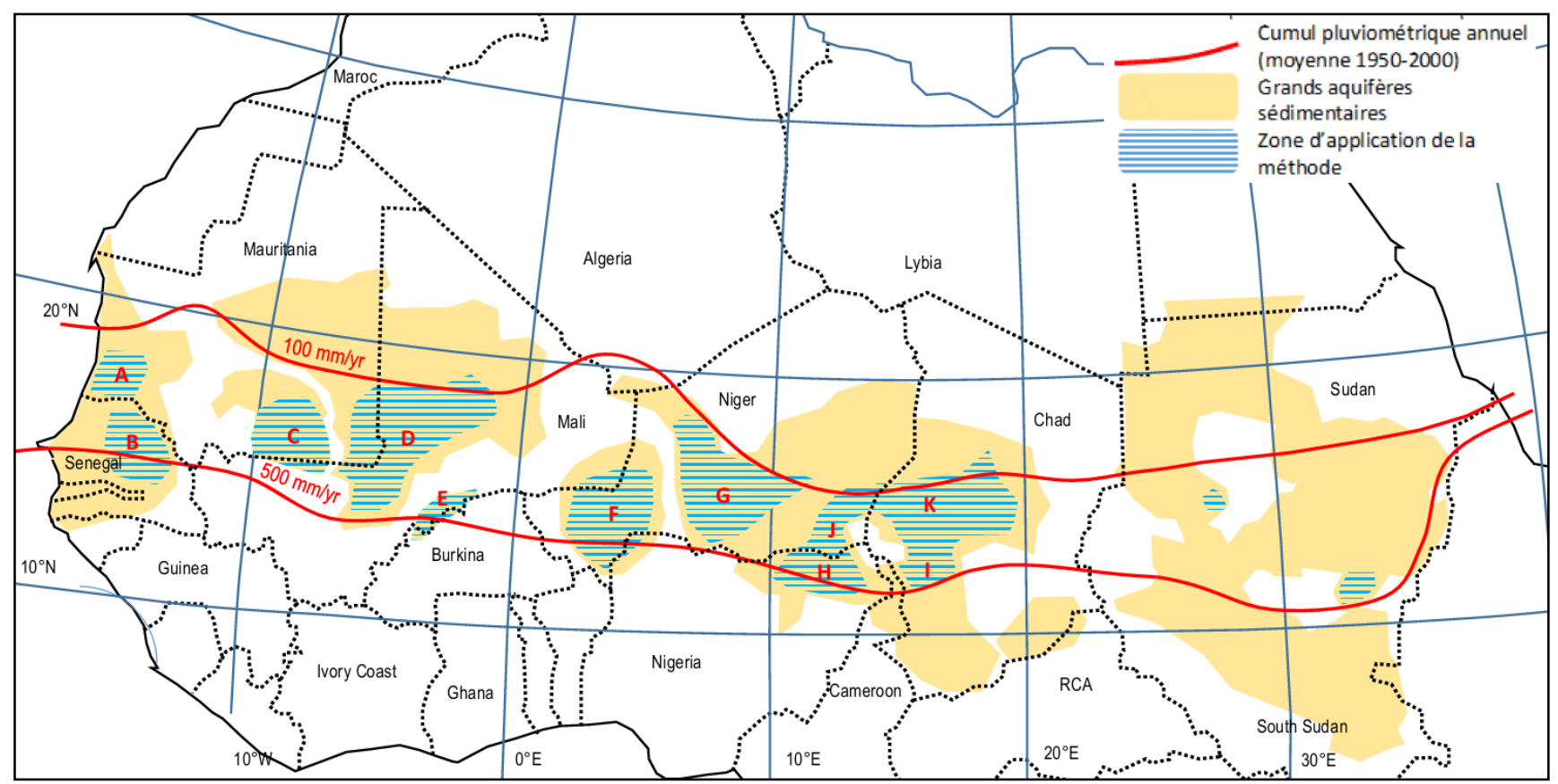

Figure 9 : Les grands aquifères sédimentaires du Sahel (en jaune) et ceux dont la carte a été dressée par télédétection (A : Trarza / B : Ferlo / C : Pelites du Hodh / D: Taoudeni / E : Gondo / F : lullemeden CT / G : lullemeden $\mathrm{Cl} / \mathrm{H}$ : Bornou / I : Chari-Baguirmi / J : Kadzell / K : Kanem - Barh el Ghazal - Batha).

\section{Références}

Bazin F., Bechir Ali B., \& Khamis Djibrine D., 2013. Systèmes d'élevage et changements climatiques au Tchad. Paris : IRAM.

Cesaro J.D., Garba I., Ickowicz A. et Toutain B., 2014. Plaidoyer pour un système d'information sur le pastoralisme au Sahel. Afrique contemporaine, n²49 pp. 90-92.

Collignon B., 2020a. A new tool for the remote sensing of groundwater table: satellite images of pastoral wells. RSP Soc. Conference.

Collignon B., 2020b. A new tool for the remote sensing of groundwater table: satellite images of pastoral wells. Open Geospatial Data, Software and Standards (Ed. Springer) 5:4. DOI 10.1186/s40965-020-00077-3.

Collignon B., \& Debris T.,1994. Entrepreneurs puisatiers du Sahel. Paris : AFVP.

Diawara M., Gangneron F.et Hiernaux P., 2014. Quelle accessibilité aux ressources pastorales du Sahel. Afrique Contemporaine, $\mathrm{n}^{\circ} 249$ - pp. 21-35.

Drake N.A., 1995. Reflectance spectra of evaporite minerals (400-2500 nm): applications for remote sensing. International Journal of Remote Sensing, 2555-2571, DOI: 10.1080/01431169508954576.

Girard C.M. \& Girard M.C., 1999. Traitement des données de télédétection. Paris : Dunod.

Krätli S. M., 2014. Accompagner la mobilité pastorale au Tchad. Afrique contemporaine, $\mathrm{n}^{\circ} 249$ - pp. 69-82 doi:10.3917/afco.249.0069.

Langlais S., Favreau G., Tapia R. \& Leduc C., 2012. La diffusion des techniques de creusement des puits et de puisage à travers la Sahara et le Sahel. Actes du colloque Mega Tchad (Naples), pp.59-83.

Langlais S., 2017. L'eau au Sahel : Expériences au Niger, en pays Touareg. Association Masnat. Ed.du Fournel.

Ministère de l'Elevage, 2016. Recensement Général de l'Elevage. N'Djamena.

Schneider J.-L., 1964. Hydrogéologie du bassin du Lac Tchad. ORSTOM.

Scoones I., 1995. Living with uncertainty: new directions in pastoral development in Africa (pp. 1-36). London: ITDG.

Valenza J., 1981. Surveillance continue de pâturages naturels de pâturages naturels sahéliens sénégalais. Revue d'élevage et de médecine vétérinaire des pays tropicaux, Vol 34, pp 81-100.

Vassolo S., Kristin Seeber K. \& Wilczok C., 2014. Groundwater Quality Investigations in the Kanem and Bahr el Ghazal regions, Chad. Hanover: BGR.

Zairi R., 2008. Etude géochimique et hydrodynamique de la nappe libre du Bassin du Lac Tchad dans les régions de Diffa (Niger oriental) et du Bornou (nord-est du Nigeria). Thèse (Univ.Montpellier II).

Zarma A.A. et Turku A., 2015. Stratigraphic Status of the Bama Beach Ridge and the Chad Formation in the Bornu Sub-Basin, Nigeria). Journal of Geology and Geophysics. 2015, 4-1. DOI 10.4172/23296755.1000192. 


\section{Titre en anglais}

\section{Contribution of optical remote sensing of pastoral wells to the mapping of groundwater in the Sahel}

\section{Légendes en anglais}

Table 1: Number of livestock in the study area (in thousands of heads)

Table 2: Potential of the method, depending on image resolution

Figure 1: Water abstraction in deep wells

Figure 2: Livestock tracks to locate pastoral wells (source: Google Earth)

Figure 3: Satellite image (Worldview) characteristic of a pastoral well in Batha or Bahr el Ghazal (source: Google Earth).

Figure 4: Interpretation of satellite images

Figure 5: Visualization of pastoral wells in the same area (Chad - West Batha) on three satellite images with an increasing spatial resolution and on an aerial photography (source: Google Earth).

Figure 6: Validation test of the method, comparing piezometric altitudes measured with two different methods.

Figure 7: Application of the remote sensing method to update, extend and improve the piezometric map of the Bornou depression (northern Nigeria). Top: Map based on direct borehole measurements (Zairi, 2008). Bottom: Map based on satellite imagery of animal tracks.

Figure 8: Statistical distribution of the depth of wells with animal tracks in Bornou (Nigeria).

Figure 9: The main sedimentary aquifers of the Sahel (in yellow) and those mapped by remote sensing (A: Trarza / B: Ferlo / C: Pelites du Hodh / D: Taoudeni / E: Gondo / F: lullemeden CT / G: lullemeden Cl / H: Bornou / I: ChariBaguirmi / J: Kadzell / K: Kanem - Barh el Ghazal - Batha).

Photos 1 and 2: water abstraction with a camel (top) and traditional acacia wood forks (bottom) in the Hadjer Lamis region of Chad (photos by Ibrahim A.Nassour).

Photo 3. Wooden pulley used by herders in the Sahel (photo Roland Tapia).

Photo 4. Accumulation of dark organic matter around a well in the Termit massif in Niger (photo Roland Tapia). 